\title{
Interaction of Green Polymer Blend of Modified Sodium Alginate and Carboxylmethyl Cellulose Encapsulation of Turmeric Extract
}

\author{
Sa-Ad Riyajan and Janthanipa Nuim \\ Department of Materials Science and Technology, Natural Product Research Center of Excellence, Faculty of Science, \\ Prince of Songkla University, Songkhla 90112, Thailand \\ Correspondence should be addressed to Sa-Ad Riyajan; saadriyajan@hotmail.com
}

Received 21 August 2013; Revised 5 October 2013; Accepted 6 October 2013

Academic Editor: Yulin Deng

Copyright (C) 2013 S.-A. Riyajan and J. Nuim. This is an open access article distributed under the Creative Commons Attribution License, which permits unrestricted use, distribution, and reproduction in any medium, provided the original work is properly cited.

Turmeric extract (tmr) loaded nanoparticles were prepared by crosslinking modified carboxylmethyl cellulose (CMC) and modified sodium alginate (SA) with calcium ions, in a high pressure homogenizer. The FTIR spectra of CMC and SA were affected by blending due to hydrogen bonding. The negative zeta potential increased in magnitude with CMC content. The smallest nanoparticles were produced with a 10:5 SA/CMC blend. Also the release rates of the extract loading were measured, with model fits indicating that the loading level affected the release rate through nanoparticle structure. The 10:5 SA/CMC blend loading with tmr and pure tmr showed a good \% growth inhibition of colon cancer cells which indicate that tmr in the presence of curcumin in tmr retains its anticancer activity even after being loaded into SA/CMC blend matrix.

\section{Introduction}

Sodium alginate (SA) and carboxymethylcellulose (CMC) are major commercial polysaccharide polymers and easily available. This is due to their advantages including low cost, biocompatibility, and biodegradability. SA consists of a-L-guluronic acid and b-D-mannuronic acid substituents and is an anionic polysaccharide, while CMC is the major commercial derivative of cellulose and is an ionic ether. These polysaccharides contain carboxylate groups $(-\mathrm{COO})$ in their backbones. The carboxylate groups $\left(-\mathrm{COO}^{-}\right)$of SA form complexes with divalent cations such as $\mathrm{Ca}^{2+}$ that cause its electrostatic crosslinking. Mixtures of SA and CMC have been studied in prior work. For example, when SA was mixed with CMC using methylenebisacrylamide (MBA) as a crosslinking agent and ammonium persulfate (APS) as an initiator, the swelling ratio of the blend decreased with increasing MBA and APS concentrations. In contrast, the swelling ratio of these hydrogels increased with the reaction temperature and with the fraction of SA. The crosslinked $\mathrm{SA} / \mathrm{CMC}$ blends exhibited a reasonable sensitivity to $\mathrm{pH}$
[1]. When both SA and CMC were blended with pullulan in an aqueous polymer solution [2], the water barrier and mechanical properties weakened significantly. The blend had comparatively weak hydrogen bonds acting on - $\mathrm{OH}$ groups, relative to pure pullulan. Crosslinked SA/CMC blends have been prepared by the casting solution method, under various gamma rays irradiation doses [3]. With increased irradiation both the gel fraction and the swelling ratio of SA/CMC blends increase, and the swelling also increases with increasing SA content. Moreover, SA/CMC blends have good mechanical and thermal properties, as well as antimicrobial activity. Microcapsules loaded with diclofenac sodium were produced from sodium carboxymethylcellulose (Na-CMC) crosslinked with $\mathrm{AlCl}_{3}$; this was used as a loaded polymer matrix that was coated with SA solution [4]. The uniformity and roundness of the formed capsules increased with the concentration of aluminum chloride, while the capsule size decreased insignificantly. The SA coating of microcapsules markedly reduced the release rate of diclofenac sodium from the core. There are many prior studies using SA as a polymer 
membrane to encapsulate a reactive agent [5-8] and corresponding reports on $\mathrm{CMC}[9,10]$. Extracts of the plant turmeric contain curcumin, which is a natural pigment [5] and potentially has antimicrobial [11], anticancer [12, 13], and wound healing activities [14]. However, curcumin is only a poorly water soluble making its therapeutic uses more difficult. Therefore, to address the solubility problem by creating water suspensions of nanoparticles, the objective of this work was to prepare turmeric-loaded nanoparticles (tmr nanoparticles) from SA/CMC blends crosslinked with $\mathrm{CaCl}_{2}$. The production of such tmr nanoparticles by using SA/CMC blends crosslinked with $\mathrm{CaCl}_{2}$ as a matrix, as potential carriers that improve both the solubility and the biological activity of tmr, has not been addressed in the prior literature. The swelling behavior for SA/CMC blends was also investigated to determine the influence of factors such as the polymer blend ratio and $\mathrm{CaCl}_{2}$ concentration on this swelling. In addition, the effects of blend ratio (SA/CMC) and salt content $\left(\mathrm{Ca}^{2+}\right)$ on the particle size 1 and zeta potential of nanoparticle were determined.

\section{Experimental}

2.1. Materials. The main materials used for this work were CMC (AR grade, Sigma-Aldrich, Seelze, Germany), SA (AR grade, Sigma-Aldrich, Seelze, Germany), and Curcumin standard (Sigma-Aldrich, China). Turmeric, dried rhizomes of C. longa L. are products of Songkhla Province, Thailand, and is purchased from Hat Yai market of Thai medicine. Turmeric extract was obtained from our laboratory. For grinding machine-assisted macerating extraction as a control for traditional extraction methods, $15 \mathrm{~g}$ of turmeric powder was extracted with a 10-fold excess volume of solvents $(\mathrm{w} / \mathrm{v})$ with $100 \mathrm{~W}$ grinding machine treatment for $1 \mathrm{~h}$, unless otherwise stated. The extraction solution was centrifuged at $5000 \mathrm{~g}$ for $20 \mathrm{~min}$, filtered through a $0.35 \mathrm{~lm}$ membrane filter and analysed by UV spectroscopy (Shimadzu UV-1601) at $428 \mathrm{~nm}(\lambda \mathrm{max})$. Deionized water was prepared using MilliQ water system (Millipore, USA). EtOH was supplied by EMSURE (Germany). Calcium chloride was purchased from Ajax Finechem Pty Ltd. (Australia). The SA and CMC were modified according to our previous work [15]. SA powder was dissolved in distilled water to get the SA solutions at $2 \%$ $(\mathrm{w} / \mathrm{w})$. The chemical modification was performed at room temperature. Initially, the $2 \% \mathrm{w} / \mathrm{w}$ SA $100 \mathrm{~g}$ was mixed with small amount of $\mathrm{H}_{2} \mathrm{O}_{2}$ (2-3 drop) and the mixture was stirred under microwave for $2 \mathrm{~min}$ to the reduction of viscosity. In case of CMC, it was modified at the same procedure with SA.

2.2. Preparation of tmr Nanoparticles. We synthesized the tmr-loaded SA/CMC nanoparticles containing $\mathrm{Ca}^{2+}$ by a simple complexation method in a high-pressure homogenizer (Pro Scientific Pro250, Monroe, CT, USA). Briefly, $0.1 \mathrm{~g}$ of tmr containing $1400 \mathrm{ppm}$ curcumin and $20 \mathrm{~g}$ of a mixture with $2 \%$ wt CMC solution and $2 \%$ SA were mixed to form a homogeneous solution under continuous stirring and then added dropwise into $50 \mathrm{~mL}$ of distilled water in the presence of $2 \% \mathrm{w} / \mathrm{w} \mathrm{CaCl}_{2}$ under stirring, to obtain the turmeric extract loaded SA/CMC nanoparticles complexed with $\mathrm{Ca}^{2+}$. Finally, the tmr nanoparticles were centrifuged at $13,000 \mathrm{rpm} / \mathrm{min}$ for $30 \mathrm{~min}$, discarding the supernatant, and resuspended with $10 \mathrm{~mL}$ of distilled water solution for further characterizations. The effect of $2 \% \mathrm{w} / \mathrm{w} \mathrm{CaCl}_{2}$ contents at 1,3 , 5 , and $7 \mathrm{~g}$ on the particle size and dynamic viscosity of $\mathrm{tmr}$ nanoparticles was investigated.

2.3. Characterization. The gel content of the SA/CMC blend was evaluated by the extraction of samples in boiling toluene for $8 \mathrm{~h}$ and the extraction of samples in boiling water for $24 \mathrm{~h}$ using Soxhlet apparatus. The extracted samples were dried in an oven at $50^{\circ} \mathrm{C}$ till constant weight. The gel fraction was calculated by the following:

$$
\% \text { Gel content }=\frac{\left(W_{0}-W_{1}\right)}{W_{0}} * 100,
$$

where $W_{1}$ and $W_{0}$ are the weights of the dried samples after extraction and before extraction, respectively. This experiment was carried out in triplicate.

The chemical structures of individual CMC, SA, and their blend were characterized by attenuated total reflection mode Fourier Transform Infrared spectrophotometer (ATR-FTIR) (Equinox 55, Bruker) with 100 scan repetitions. The nanoparticle sizes in water suspension were determined with a Zeta seizer (DLS using DLS-ZP/Particle Sizer Nicomp 380 ZLS). The nanoemulsion samples were loaded into $1 \mathrm{~cm}^{3}$ cuvettes and put into a thermostat regulated chamber. Dynamic light scattering was observed at a $90^{\circ}$ angle.

The SA/CMC blend solution at different blend ratios $(50.0 \mathrm{~mL})$ was immediately transferred into the beak cup reservoir of the rotational viscometer. The data of the viscometer output parameters started $1 \mathrm{~min}$ after the experiment onset. The alters of dynamic viscosity $(\eta)$ and torque were measured at different temperatures using a digital rotational viscometer Brookfield DV-II+ PRO (Brookfield Engineering Labs Inc., Middleboro, MA, USA). At the spindle rotational speed of $180 \mathrm{rpm}$, the shear rate equaled $237.6 \mathrm{~s}^{-1}$ and this experiment was carried out in triplicate. The morphology of dry tmr nanoparticles was investigated by AFM OCA15EC (Data Physics). Transmission Electron Microscopy (TEM) with JEOL JEM-2010 was also used to study the morphology. The dispersed tmr nanoparticles were diluted approximately 400-fold from their original concentration, with distilled water. They were then placed on a 400 mesh grid and dried overnight in a desiccator before characterization.

The particle size of our nanoparticle system dispersed in water was obtained by zeta potential measurements (DLSZP/Particle Sizer Nicomp 380 ZLS).

The efficiency of tmr entrapment in the SA/CMC matrix was calculated from the ratio between the initial mass of tmr to be encapsulated and its mass in the final product. In this case, a known amount of tmr nanoparticles (ca. $12 \mathrm{mg}$ ) was dispersed in a 50/50 EtOH/ $\mathrm{H}_{2} \mathrm{O}$ mixture and stirred for 2 days at room temperature. Subsequently, the suspension was filtered, and the content of dissolved tmr in the 50/50 $\mathrm{EtOH} / \mathrm{H}_{2} \mathrm{O}$ mixture solution was determined by UV-visible spectrometry (Shimadzu UV-1601) at $428 \mathrm{~nm}(\lambda \max )$. At 
definite intervals of time, an aliquot $(5 \mathrm{~mL})$ was taken for the analysis of tmr in tmr nanoparticles. Experiments were performed thrice. The cumulative release of $\mathrm{tmr}$ from $\mathrm{tmr}$ nanoparticles was estimated from the following:

$$
\% \text { Cumulative tmr from tmr nanoparticles }=\frac{M_{t}}{M_{\infty}} \text {. }
$$

$M_{t}$ is cumulative amounts of drug released at time $t$, while $M_{\infty}$ is the cumulative amounts of drug released at time $t$ and at infinite time.

The release results were evaluated by using an empirical equation to estimate the value of $n$ as follows:

$$
\frac{M_{t}}{M_{\infty}}=K t^{n} \text { or } \log \left(\frac{M_{t}}{M_{\infty}}\right)=\log (K)+n \log (t),
$$

where $M_{t} / M_{\infty}$ is the released fraction at time $t, n$ is the release exponent, and $K$ is the release factor. From the slope and intercept of the plot of $\log \left(M_{t} / M_{\infty}\right)$ against $\log (t)$, kinetic parameters $n$ were calculated.

In addition, the anticancer activity of the tmr nanoparticles was assessed in vitro with select cell lines, observed by methyl thiazol tetrazolium bromide (MTT) assay.

The HT-29 (human colon adenocarcinoma) cells line was grown in Dulbecco's Modified Eagle Medium (DMEM) supplemented with $10 \%$ fetal bovine serum (FBS). Cells were seeded in 96-well plates (3500 cells/well) and allowed to adhere for 1 day at $37^{\circ} \mathrm{C}$ with $5 \% \mathrm{CO}_{2}$ in a fully humidified incubator. Then $100 \mu \mathrm{L}$ of $25 \mu \mathrm{L} / \mathrm{mL}$ sample in medium were dispensed into wells of the cell plates and incubated further for 3 days. After removal of the sample medium, the cells were topped up with $200 \mu \mathrm{L}$ D-MEM medium and incubated. After $72 \mathrm{~h}$ cells were fixed with cold $40 \%$ trichloroacetic acid, kept in $4^{\circ} \mathrm{C}$ for $1 \mathrm{~h}$, and washed with tap water. The cells were assessed with sulforhodamine assay, and the absorbance was measured at $492 \mathrm{~nm}$ using a microplate reader. The calculated results describe the inhibition of cell growth as compared with control (cells in media without tmr):

$$
\% \text { inhibition }=\frac{\text { OD control }- \text { OD sample }}{\text { OD control }} \times 100 \text {. }
$$

Here, OD is the absorbance at $492 \mathrm{~nm}$.

\section{Results and Discussion}

3.1. Interaction between CMC and SA and Curcumin in tmr. The effect of SA on the gel content and swelling ratio of SA/CMC in water are presented in Figure 1.

$\mathrm{SA}$ at $2 \% \mathrm{w} / \mathrm{w}$ was blended with CMC to decrease the dissolution of SA in water. This is due to hydrogen bonding between SA and CMC as shown in Figure 1(a) and Figure 1(b), respectively. The gel content of the $10: 2.5$ SA/CMC blend was $80 \%$. These results indicate a good interaction between $\mathrm{CMC}$ and $\mathrm{SA}$ as expected [1]. When concentration of $\mathrm{CMC}$ was increased in the mixing ratios $10: 5,10: 10$, and $10: 15 \mathrm{SA} / \mathrm{CMC}$ blend the gel content of the samples was a constant 90\%. However, at 10:25 SA/CMC blend, the gel content decreased due to dissolution in water of SA. The unbalance of SA and CMC amounts reduced their mutual interaction [1]. After the addition of tmr in SA/CMC blend, the hydrogen interaction between hydroxyl group from curcumin in tmr and carboxylic group of SA and $\mathrm{CMC}$ as represented in Figure 2(b). These phenomena result in the controlling release of tmr from bead and the more details were discussed in Section 3.6.

The possible crosslinking between $\mathrm{Ca}^{2+}$ and carboxylic groups of CMC and carboxylic group of SA are shown in Figure 3(a) and Figure 3(b), respectively. The influence of $\mathrm{CaCl}_{2}$ on the swelling ratio of the 10:5 SA/CMC blend is displayed in Figure 1(b). Increasing $\mathrm{CaCl}_{2}$ decreases the swelling ratio of SA/CMC due to contraction of SA/CMC blend $[16,17]$. With $\mathrm{CaCl}_{2}$ concentration at $0.2,0.4,1$, and $2 \% \mathrm{w} / \mathrm{w}$, the swelling ratio of 10:5 SA/CMC blend was 50, 40,40 , and $30 \%$, respectively. The $\mathrm{CaCl}_{2}$ in polymer blend solution reacts with carboxyl functional groups of $\mathrm{SA}$ to form a crosslinked SA, restricting movement of the polymer chains and their ability to swell [18]. After adding $\mathrm{CaCl}_{2}$ in the $\mathrm{SA} / \mathrm{CMC} / \mathrm{tmr}$ mixture, tmr nanoparticles are formed, and a schematic conceptual model of a nanoparticle is shown in Figure 3(c). The tmr is dispersed in the polymer matrix of SA/CMC blend, crosslinked with $\mathrm{CaCl}_{2}$. CMC forms aggregate particles with $\mathrm{CaCl}_{2}$, while the carboxylic groups of SA are ionically crosslinked with $\mathrm{Ca}^{2+}$.

3.2. FTIR Study. In order to investigate the chemical crosslinking reaction between $\mathrm{Ca}^{2+}$ and carboxylic groups of SA, FTIR was used and the results are shown in Figure 4. FTIR in 2000-400 $\mathrm{cm}^{-1}$ range showed stretching of carboxyl groups of SA at 1607 and $1424 \mathrm{~cm}^{-1}$, assigned to symmetric and antisymmetric $\mathrm{COO}$ stretching vibrations of the free carboxylate groups $[8,19,20]$. The absorption band of CMC homopolymer appears at $2983 \mathrm{~cm}^{-1}$ referring to C$\mathrm{H}$ stretching and $\mathrm{C}-\mathrm{O}$ stretching of the ether group of the carboxymethylation of cellulose and at $1187 \mathrm{~cm}^{-1}$ assigned to the ether linkage [1,4- $\beta$-d-glucoside] of cellulose [21]. There are two further strong bands. The first strong FTIR absorption band at $3223 \mathrm{~cm}^{-1}$ is due to $\mathrm{O}-\mathrm{H}$ stretching of the nonsubstituted hydroxyl groups of cellulose. The second strong absorption band seen at $821 \mathrm{~cm}^{-1}$ is due to the $\mathrm{C}-\mathrm{O}$ stretching of alcohol. The main ATIR bands of SA at 1620 and $1416 \mathrm{~cm}^{-1}$ are assigned to asymmetric and symmetric stretching peaks of carboxylate salt groups. In addition, the FTIR bands around 1320, 1131, 1022, and $949 \mathrm{~cm}^{-1}$ are referred to $\mathrm{C}-\mathrm{O}$ stretching, $\mathrm{C}-\mathrm{C}$ stretching, $\mathrm{C}-\mathrm{O}-\mathrm{C}$ stretching, and $\mathrm{C}-\mathrm{O}$ stretching, respectively, of the saccharide structure. The FTIR spectra of CMC and SA were affected by blending due to hydrogen bonding. Similar findings have been reported for the addition of lactose to alginic acid [20].

\subsection{Parameters Affecting Nanoparticle Size}

3.3.1. Influence of CMC on Particle Size. The effect of CMC content on the particle size of tmr nanoparticles is plotted in Figure 5(a). The average capsule size increased with CMC content. With CMC contents from $0.2 \%$ to be $0.4 \%$ 


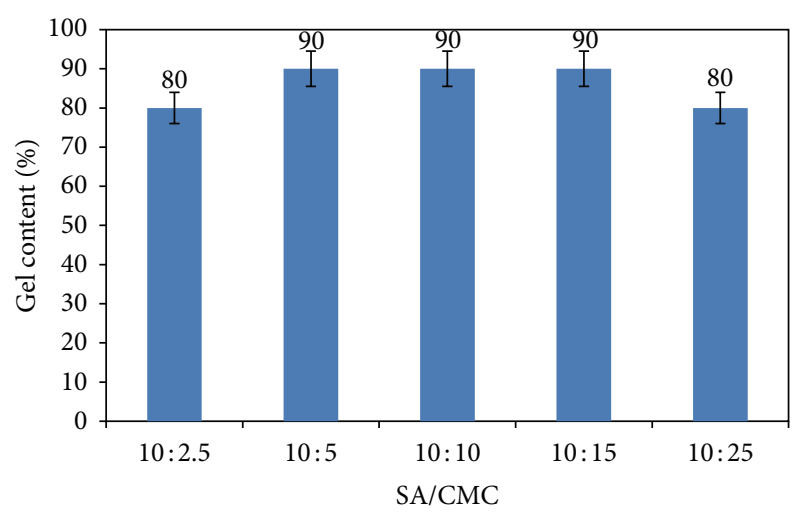

(a)

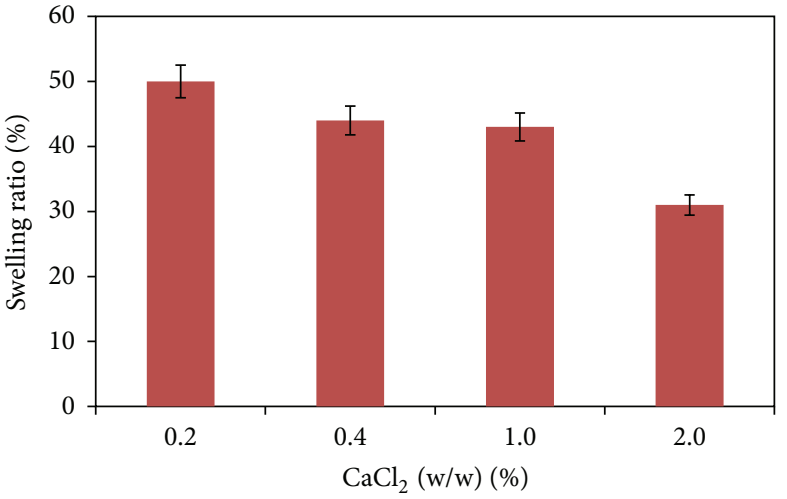

(b)

FIGURE 1: Effect of polymer blend ratio on the (a) gel content and (b) effect of $\mathrm{CaCl}_{2}$ concentration on the swelling ratio of SA/CMC blends in water.

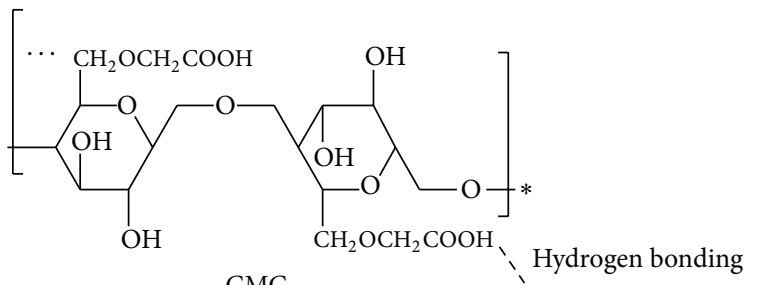

CMC

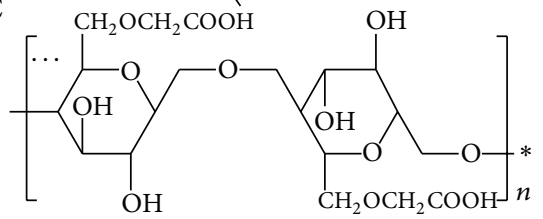

CMC

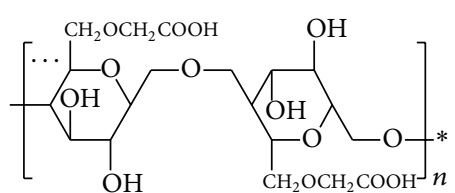

$\mathrm{CMC}$<smiles>[Te+2]=[Pt+2]</smiles>
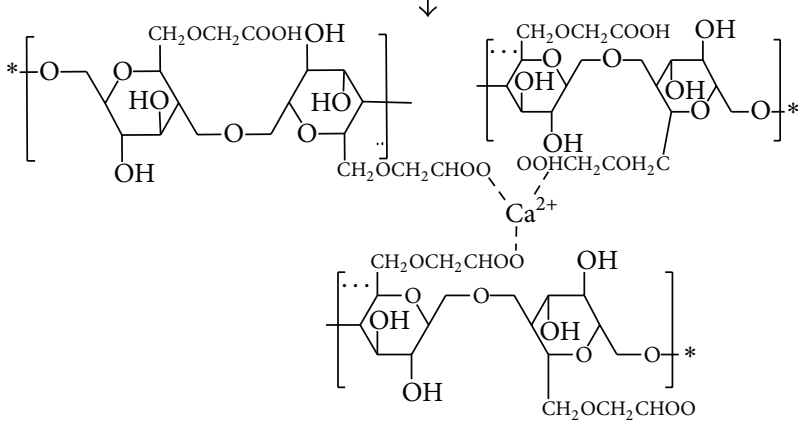

Complex between $\mathrm{CMC}$ and $\mathrm{Ca}^{2+}$

(b)

Figure 2: (a) Possible hydrogen bonding between CMC and SA and (b) interaction between $\mathrm{Ca}^{2+}$ and SA/CMC blend.

in the blend the average tmr nanoparticle size was about $380 \mathrm{~nm}$. The smallest nanoparticles were produced with a $10: 5 \mathrm{SA} / \mathrm{CMC}$ blend. However, with 1\% CMC the average particle size was about $820 \mathrm{~nm}$, so the CMC concentration of the solutions strongly affected the particle size. From the literature, the capsule size is controlled by dynamic viscosity and consequently by the polymer concentration [22]. The dynamic viscosities determined for CMC solutions are shown in Figure 5(b), which clearly correlates with the observed particle sizes.

3.3.2. Effect of SA on Particle Size. The influence of SA on the tmr nanoparticle size is displayed in Figure 6(a). The size increased with SA concentration [22]. The lowest particle size was observed at $0.2 \% \mathrm{SA}$. The dynamic viscosities of
SA solutions are shown in Figure 6(b) and are considerably higher than those for CMC solutions.

3.3.3. Effect of $\mathrm{CaCl}_{2}$ on Particle Size of tmr Nanoparticle. Figure 7 (a) shows the effect of $\mathrm{CaCl}_{2}$ concentration on the tmr nanoparticle size made from 10:5 SA/CMC blend. The highest particle size was found at $5 \mathrm{~g}$ of $1 \% \mathrm{CaCl}_{2}$. At high $\mathrm{CaCl}_{2}$ salt concentration, the $\mathrm{CMC}$ molecule chains lose their flexibility; the hydrodynamic size of the molecules diminishes, producing an agglomeration of polymer chains, and resulting in the decrease of nanoparticle size [23].

Adding $\mathrm{CaCl}_{2}$ causes crosslinking between carboxylic groups of $\mathrm{CMC}$ and $\mathrm{CaCl}_{2}$ as shown in Figure 3(a). Figure $7(\mathrm{~b})$ is a plot of $2 \% \mathrm{w} / \mathrm{w} \mathrm{CaCl}_{2}$ concentration that affects the dynamic viscosity of SA/CMC blend solution. 


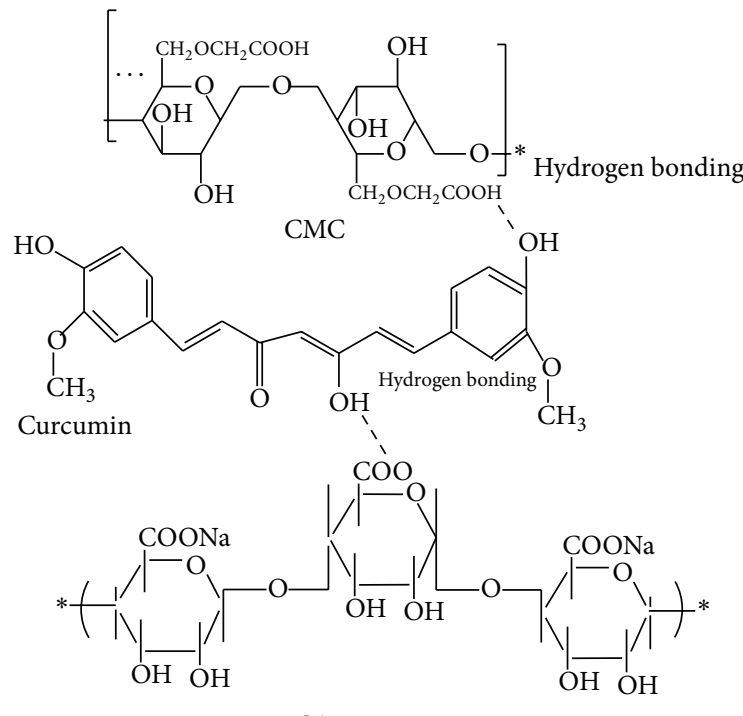

SA

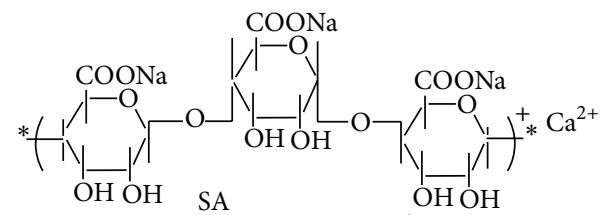

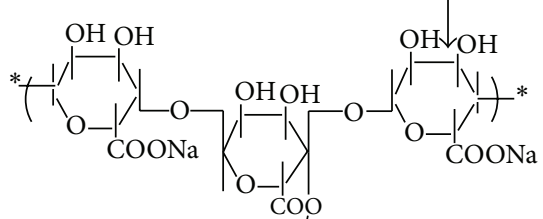

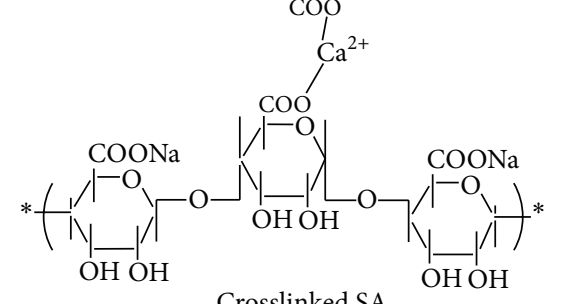

Crosslinked SA

(a)

(b)

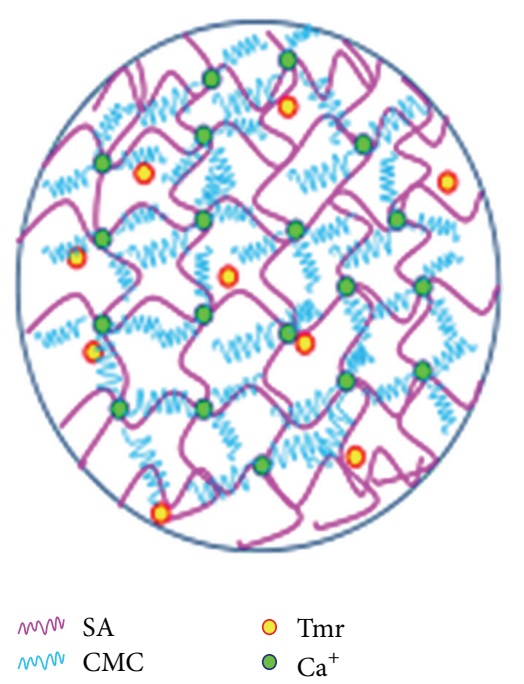

(c)

FIGURE 3: (a) Ionic crosslinking of CMC blend in SA/CMC blend with $\mathrm{Ca}^{2+}$, (b) ionic crosslinking of SA blend in SA/CMC blend with Ca ${ }^{2+}$, and (c) a conceptual schematic model of tmr nanoparticle.

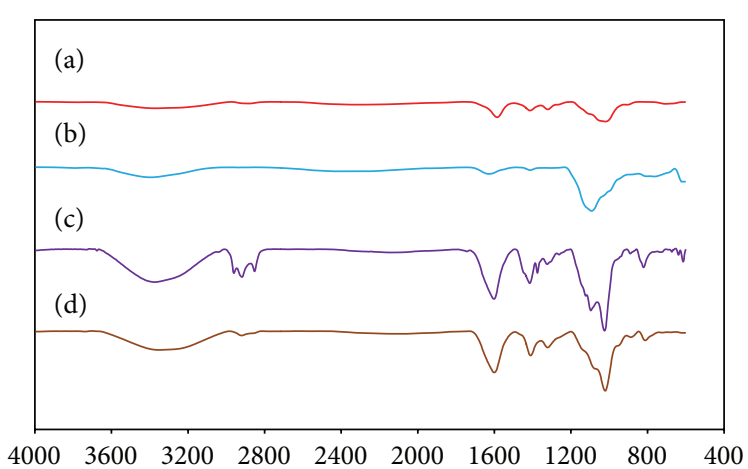

Figure 4: ATR-FTIR spectra of (a) CMC, (b) SA, (c) 10:5 SA/CMC blend, and (d) 10:5 CMC SA/CMC blend crosslinked with Ca ${ }^{2+}$. 


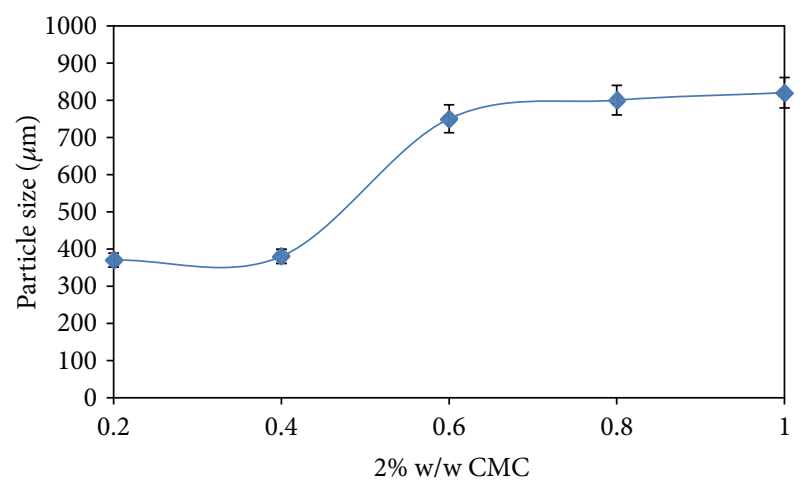

(a)

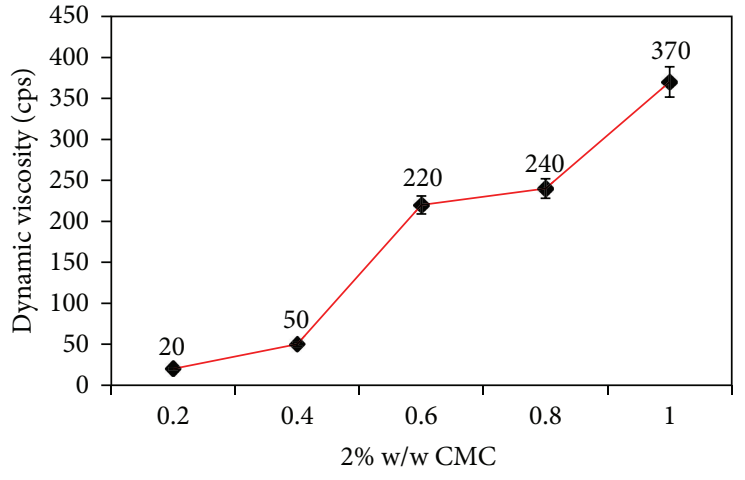

(b)

Figure 5: Effect of CMC on (a) particle size and (b) dynamic viscosity of SA/CMC blend crosslinked with $\mathrm{Ca}^{2+}$.

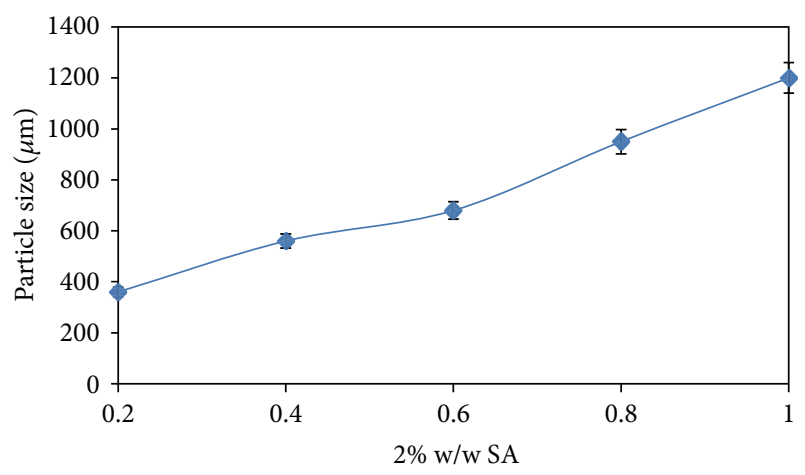

(a)

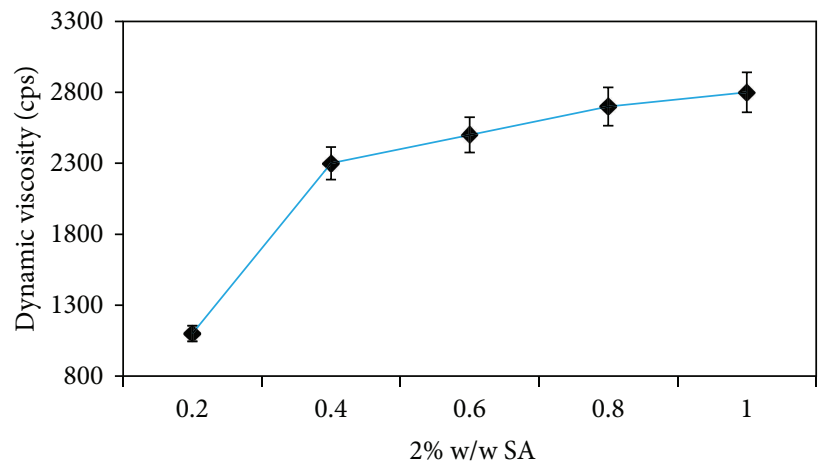

(b)

Figure 6: Effect of SA on (a) particle size and (b) dynamic viscosity of SA/CMC blend crosslinked with $\mathrm{Ca}^{2+}$.

The dynamic viscosity dramatically decreased with $\mathrm{CaCl}_{2}$ concentration, because $\mathrm{CaCl}_{2}$ promoted aggregate formation of CMC and SA. Khaled and Abdelbaki [23] studied the effects of ions from calcium chloride, sodium chloride, and potassium chloride on the rheological and electrokinetic properties of dilute $0.50 \%$ sodium carboxymethylcellulose (Na-CMC) dispersions in a coaxial cylinder viscometer over a wide range of shear rates $\left(0\right.$ to $\left.600 \mathrm{~s}^{-1}\right)$. The ions reduce the consistency index, and the rheological parameters of CMC solutions in a saline medium are quasi-stable [23].

3.4. Zeta Potential Study. The effects of CMC content on the zeta potential of nanoparticles are shown in Figure 8(a). The negative zeta potential increased in magnitude with CMC content. The negative charge of $\mathrm{CMC}$ and $\mathrm{SA}$ is due to the dissociation of $\mathrm{Na}^{+}$from carboxylic groups [24]. The influence of $\mathrm{CaCl}_{2}$ on the Zeta potential are illustrated in Figure $8(\mathrm{~b})$, showing that the magnitude of negative zeta potential decreased with the salt concentration. This may be due to the neutralization between the negative charges from carboxylic group of SA/CMC and $\mathrm{Ca}^{2+}$ ions, leading to a reduction of zeta potential [23].
3.5. Morphology by TEM. The shapes and sizes of tmr nanoparticles were observed under TEM, and the results are shown in Figure 9. The tmr nanoparticles, with 10:5 $\mathrm{SA} / \mathrm{CMC}$ blend as the polymer matrix crosslinked in the presence of $3 \mathrm{~g} \mathrm{CaCl}_{2}$, had an average size of about $550 \pm$ $20 \mathrm{~nm}$, as shown in Figure 9(a). When $2 \% \mathrm{w} / \mathrm{w} \mathrm{CaCl}_{2}$ content in medium increased from 3 to $7 \mathrm{~g}$, the mean particle size decreased to $350 \pm 20 \mathrm{~nm}$ due to increased crosslinking density; see Figure 9(b). The shape of tmr nanoparticles change from spherical into rectangular shapes as shown in Figure 9(b) due to increasing concentration of ions [25]. The previous study of the influence of silver concentration on the change in shape of Ag ions was found. For example, at $1.2 \mathrm{mM}$ silver nitrate solution, no significant shape and composition occur in silver templates. The shape of this product was found to be a spherical shape and few numbers of ringling particles. When the concentration of $1.2 \mathrm{mM}$ silver nitrate solution increased from $1.2 \mathrm{mM}$ to $4.8 \mathrm{mM}$, the shape of silver was decahedral. This size trend was also seen in the ZetaSizer measurements, but the actual sizes differ due to drying shrinkage of capsules $[8,26]$. The tmr nanoparticles imaged had various shapes including spherical, egg, and rectangular shapes. 


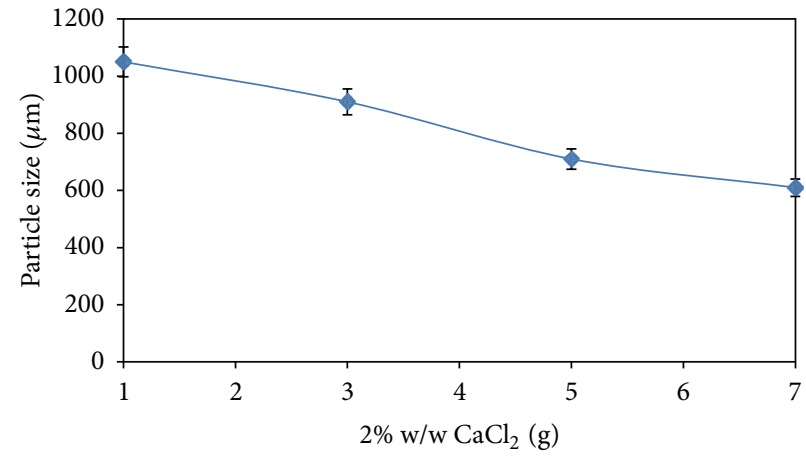

(a)

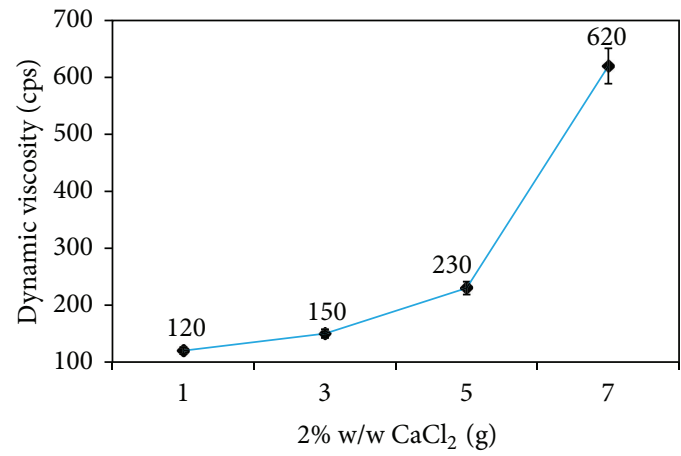

(b)

Figure 7: Effect of $\mathrm{CaCl}_{2}$ on (a) particle size and (b) dynamic viscosity of 10:5 SA/CMC blend crosslinked with Ca ${ }^{2+}$.

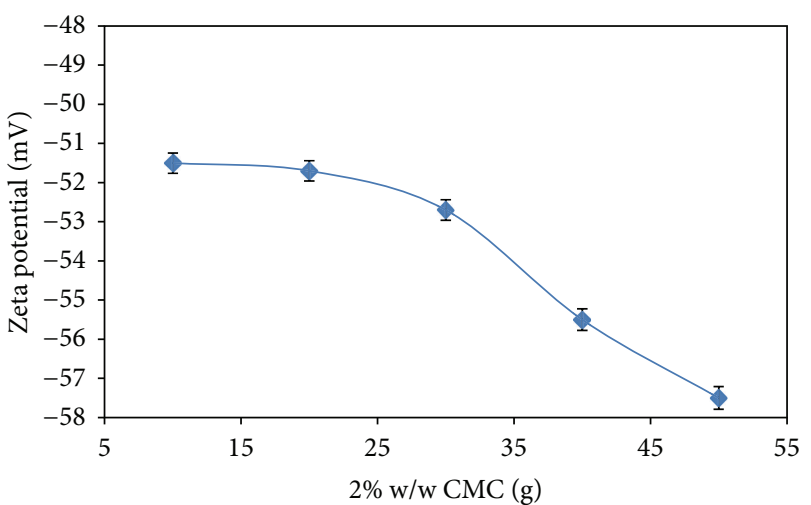

(a)

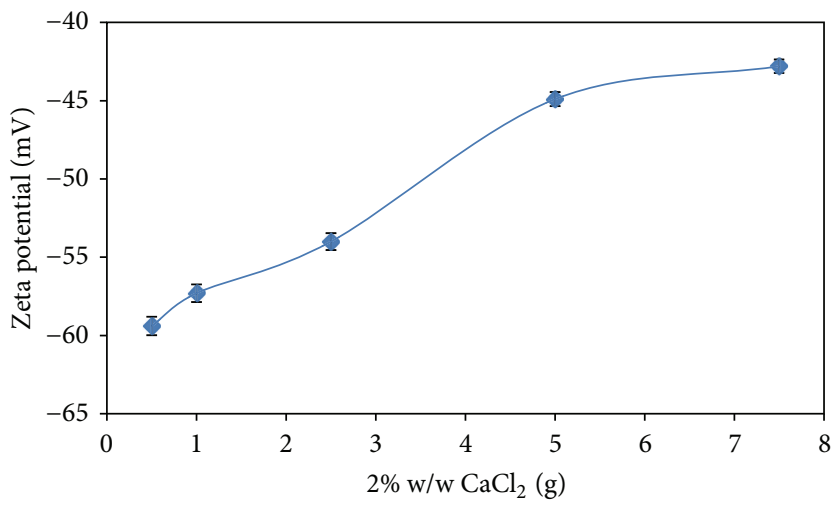

(b)

FigurE 8: Effect of (a) CMC (g) and (b) $\mathrm{CaCl}_{2}$ on the zeta potential of nanocapsules of SA/CMC blend crosslinked with $\mathrm{Ca}^{2+}$.

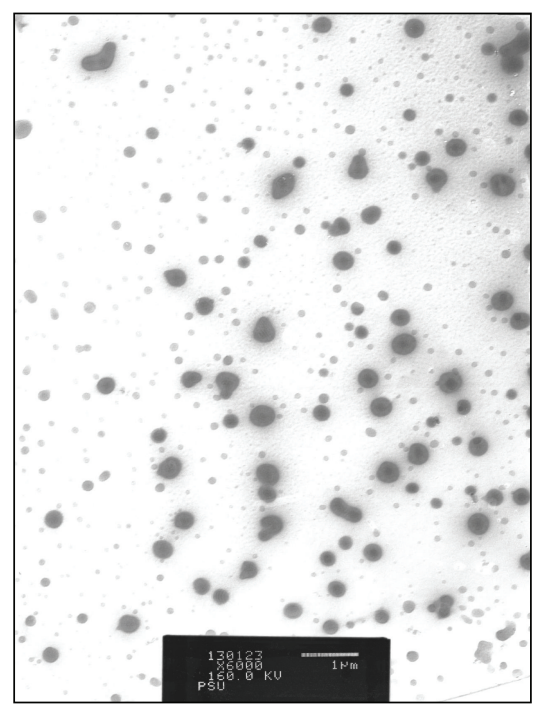

(a)

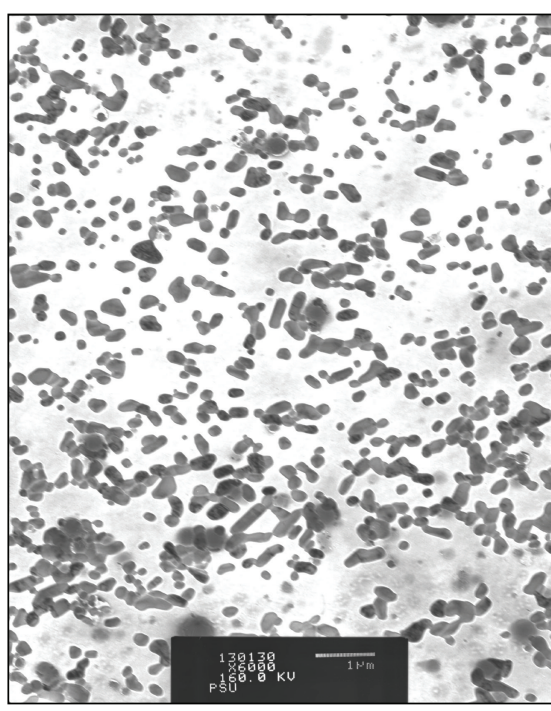

(b)

FIGURE 9: TEM images of crosslinked 10:5 SA/CMC blend obtained with (a) $3 \mathrm{~g}$ and (b) $5 \mathrm{~g} 5 \% \mathrm{w} / \mathrm{w} \mathrm{CaCl}_{2}$. 


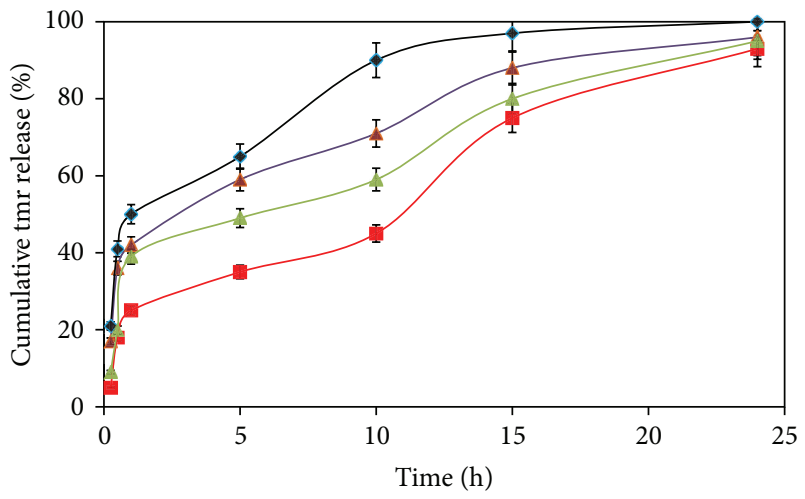

(a)

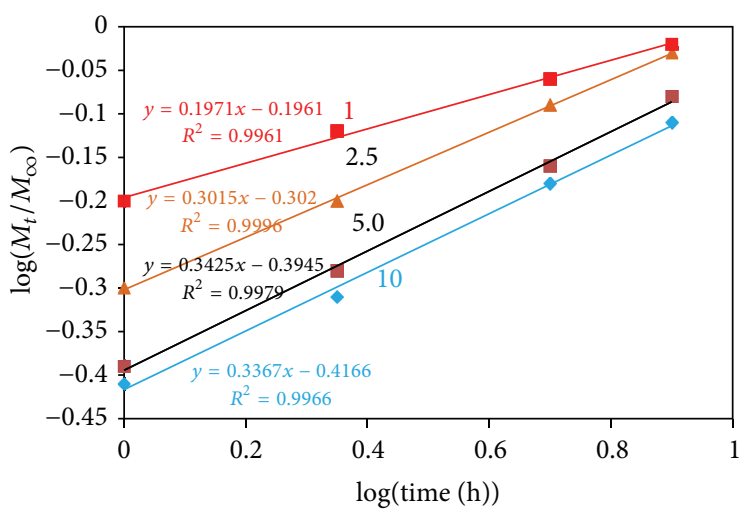

(b)

FIGURE 10: Effect of tmr loading on release rate of tmr from nanoparticles (a) and (b) relationship between $\log \left(M_{t} / M_{\alpha}\right)$ and $\log (t)$.

3.6. Release of tmr Loading. The effect of tmr content on the kinetics of tmr release is depicted in Figure 10(a). Higher tmr contents clearly give higher release rates. This might be due to the tmr easing the penetration of liquid as well as tmr diffusion within the nanoparticle, through effects on its microstructure, especially porosity. A prior study of the encapsulation of neem with poly(vinyl alcohol) (PVA)/SA blend as polymeric membrane showed that the rate of $\mathrm{tmr}$ release increased with neem content due to thermodynamics forces [8].

In power law fits of diffusion data, the exponent $\mathrm{n}$ is an indication of the rate limiting mechanism. An $n=0.5$ indicates that the release is Fickian diffusion controlled [8], while $n=1$ represents zero-order release. Values of $n$ between these ideal cases indicate potentially a reactive agent release mechanism, or non-Fickian diffusion, or chain relaxation controlled release. From the plots of $\log \left(M_{t} / M_{\infty}\right)$ against $\log (t)$, release exponents $(n)$ were calculated as shown in Figure $10(\mathrm{~b})$. The $n$ value increased as a function of $\mathrm{tmr}$ in polymer blend and then remains a constant value. For the sample with $10 \mathrm{gtmr}$ a regression fit gave approximately $n=$ 0.336 , so the release was not Fickian diffusion controlled, and with $5 \mathrm{~g}$ tmr the results $n=0.342$ were closely similar. The dependence of the $n$ value on tmr concentration suggests that the particle structure is affected in this range of tmr contents.

3.7. Cell Uptake. The cellular uptake by normal and cancer cells of nanoparticles with various polymer blend ratios was quantified, using ethanol extraction and spectrophotometric observation at $492 \mathrm{~nm}$.

The 10:5 SA/CMC blend without tmr showed $80 \%$ growth inhibition of cancer cells from human colon adenocarcinoma. This might be due to interaction of carboxylic group from SA/CMC and colon cell cancer. After the addition of tmr in polymer blend, the 10:5 SA/CMC blend loading with tmr showed comparable effect on colon cancer cells which indicate that tmr in the presence of curcumin retains activity even its anticancer after being loaded into polymer blend matrix. This result was in agreement with the previous work [27].
TABLE 1

\begin{tabular}{lc}
\hline$\%$ tmr & \% Inhibition \\
\hline 0 & $80.821 \pm 0.384$ \\
1 & $90.531 \pm 0.544$ \\
2.5 & $97.253 \pm 0.651$ \\
5 & $97.602 \pm 0.503$ \\
10 & $97.832 \pm 0.542$ \\
Pure 2.5\%tmr & $98.544 \pm 0.522$ \\
\hline
\end{tabular}

The $\%$ growth inhibition of a cancer cell line in vitro was 97-98\%, as shown in Table 1. It is known that curcumin, a hydrophobic polyphenol derived from turmeric, has anticarcinogenic activity [5]. Our nanoparticles were in the optimal below $800 \mathrm{~nm}$ size range for drug delivery applications [5]. The efficiency of SA/CMC blend loaded with tmr in \% growth inhibition of colon cancer cell showed lower than that of unloaded tmr owing to its encapsulation in polymer matrix as shown in Figure 3(c).

\section{Conclusions}

Nanoparticles loaded with turmeric extract were successfully prepared from SA/CMC crosslinked with $\mathrm{CaCl}_{2}$, in a pressure homogenizer. Increasing $\mathrm{CaCl}_{2}$ decreases the swelling ratio of SA/CMC due to chemical interaction between $\mathrm{Ca}^{2+}$ and hydroxyl group SA/CMC blend matrix and confirmed by FTIR. Moreover, the hydrogen interaction between hydroxyl group from curcumin in tmr and carboxylic group of SA and $\mathrm{CMC}$ after the addition of tmr in SA/CMC blend. The nanoparticle size dramatically increased with SA and CMC concentrations but decreased with calcium salt concentration, because the salt was a crosslinking agent. The smallest nanoparticles were produced with a 10:5 SA/CMC blend. The tmr nanoparticles imaged had various shapes including spherical, egg, and rectangular shapes depending on tmr concentration. Higher tmr contents clearly give higher release rates. The $n$ value increased with increasing tmr content in polymer blend. The release pattern was not Fickian diffusion 
controlled. The dependence of the $n$-value on tmr concentration was found. The 10:5 SA/CMC blend loading with tmr and pure tmr showed comparable effect on colon cancer cells which indicate that $\mathrm{tmr}$ in the presence of curcumin in tmr retains its anticancer activity even after being loaded into polymer blend matrix. The \% growth inhibition of a cancer cell line in vitro was $97-98 \%$.

\section{Acknowledgments}

The authors would like to acknowledge the financial support from the National Nanotechnology Center (NANOTEC) and the National Science and Technology Development Agency (NSTDA), Thailand (P-11-00981), to Prince of Songkla University and Natural Product Research Center of Excellence Faculty of Science, Prince of Songkla University.

\section{References}

[1] A. Pourjavadi, S. Barzegar, and G. R. Mahdavinia, "MBAcrosslinked Na-Alg/CMC as a smart full-polysaccharide superabsorbent hydrogels," Carbohydrate Polymers, vol. 66, no. 3, pp. 386-395, 2006

[2] Q. Tong, Q. Xiao, and L.-T. Lim, "Preparation and properties of pullulan-alginate-carboxymethylcellulose blend films," Food Research International, vol. 41, no. 10, pp. 1007-1014, 2008.

[3] S. M. Ibrahim and K. M. El Salmawi, "Preparation and properties of Carboxymethyl Cellulose (CMC)/Sodium alginate (SA) blends induced by gamma irradiation," Journal of Polymers and the Environment, vol. 21, pp. 520-527, 2012.

[4] E. A. Hosny and A. A.-R. M. Al-Helw, "Effect of coating of aluminum carboxymethylcellulose beads on the release and bioavailability of diclofenac sodium," Pharmaceutica Acta Helvetiae, vol. 72, no. 5, pp. 255-261, 1998.

[5] A. F. Martins, P. V. A. Bueno, E. A. M. Almeida S, F. H. A. Rodrigues, A. F. Rubira, and E. C. Muniz, "Characterization of N-trimethyl chitosan/alginate complexes and curcumin release," International Journal of Biological Macromolecules, vol. 57, pp. 174-184, 2013.

[6] R. Hurteaux, F. Edwards-Lévy, D. Laurent-Maquin, and M.-C. Lévy, "Coating alginate microspheres with a serum albuminalginate membrane: application to the encapsulation of a peptide," European Journal of Pharmaceutical Sciences, vol. 24, no. 2-3, pp. 187-197, 2005.

[7] A. Blandino, M. Macías, and D. Cantero, "Formation of calcium alginate gel capsules: influence of sodium alginate and $\mathrm{CaCl}_{2}$ concentration on gelation kinetics," Journal of Bioscience and Bioengineering, vol. 88, no. 6, pp. 686-689, 1999.

[8] S.-A. Riyajan and J. T. Sakdapipanich, "Characterization of biodegradable semi-interpenetrating polymer based on poly(vinyl alcohol) and sodium alginate containing natural neem (Azadirachta indica) for controlled release application," Polymer International, vol. 59, no. 8, pp. 1130-1140, 2010.

[9] N. Pahimanolis, A. Salminen, Penttilä et al., "Nanofibrillated cellulose/carboxymethyl cellulose composite with improved wet," Cellulose, vol. 20, no. 3, pp. 1459-1468, 2013.

[10] K. Varaprasad, K. Vimala, S. Ravindra, N. Narayana Reddy, G. Venkata Subba Reddy, and K. Mohana Raju, "Fabrication of silver nanocomposite films impregnated with curcumin for superior antibacterial applications," Journal of Materials Science, vol. 22, no. 8, pp. 1863-1872, 2011.

[11] K. S. Parvathy, P. S. Negi, and P. Srinivas, "Antioxidant, antimutagenic and antibacterial activities of curcumin- $\beta$-diglucoside," Food Chemistry, vol. 115, no. 1, pp. 265-271, 2009.

[12] R. Prasanna, H. Chinnakonda Chandramoorthy, P. Ramaiyapillai, and D. Sakthisekaran, "In vitro evaluation of anticancer effect of Cassia auriculata leaf extract and curcumin through induction of apoptosis in human breast and larynx cancer cell lines," Biomedicine and Preventive Nutrition, vol. 1, no. 2, pp. 153-160, 2011.

[13] J. Chen, G. Wang, L. Wang, J. Kang, and J. Wang, "Curcumin p38-dependently enhances the anticancer activity of valproic acid in human leukemia cells," European Journal of Pharmaceutical Sciences, vol. 41, no. 2, pp. 210-218, 2010.

[14] X. Li, K. Nan, L. Li, Z. Zhang, and H. Chen, "In vivo evaluation of curcumin nanoformulation loaded methoxy poly(ethylene glycol)-graft-chitosan composite film for wound healing application," Carbohydrate Polymers, vol. 88, no. 1, pp. 84-90, 2012.

[15] S. Riyajan and J. Nuim, "Preparation and characterization of coating turmeric-coconut oil nanoemulsion with modified sodium alginate," Advanced Materials Letters, vol. 19, pp. 741745, 2013.

[16] J. S. Yang, Q. Q. Zhou, and W. He, "Amphipathicity and selfassembly behavior of amphiphilic alginate esters," Carbohydrate Polymers, vol. 92, pp. 223-227, 2013.

[17] Z. Wang, Z. Fu, and C. Ye, "Recovery of nickel from aqueous samples with water-soluble carboxyl methyl cellulose-acetone system," Journal of Hazardous Materials, vol. 170, no. 2-3, pp. 705-710, 2009.

[18] H. U. Rehman, A. Aman, A. Silipo, S. A. U. Qader, A. Molinaro, and A. Ansari, "Degradation of complex carbohydrate: immobilization of pectinase from Bacillus licheniformis KIBGE-IB21 using calcium alginate as a support," Food Chemistry, vol. 139, no. 1-4, pp. 1081-1086, 2013.

[19] A. Mahmood, S. Bano, S. G. Kim, and K. H. Lee, "Watermethanol separation characteristics of annealed SA/PVA complex membranes," Journal of Membrane Science, pp. 360-367, 2012.

[20] A. Mahmood, S. Bano, S. G. Kim, and K. H. Lee, "Flocculation performance of trimethyl quaternary ammonium salt of ligninsodium alginate polyampholyte," BioResources, vol. 8, no. 3, pp. 3544-3555, 2013.

[21] R. Liu and D. Zhao, "Synthesis and characterization of a new class of stabilized apatite nanoparticles and applying the particles to in situ $\mathrm{Pb}$ immobilization in a fire-range soil," Chemosphere, vol. 91, pp. 594-601, 2013.

[22] J. Liu, L. Xu, C. Liu et al., "Preparation and characterization of cationic curcumin nanoparticles for improvement of cellular uptake," Carbohydrate Polymers, vol. 90, no. 1, pp. 16-22, 2012.

[23] B. Khaled and B. Abdelbaki, "Rheological and electrokinetic properties of carboxymethylcellulose-water dispersions in the presence of saltsInternational," Journal of Physical Science, vol. 7, pp. 1790-1798, 2012.

[24] P. Bacchin, J.-P. Bonino, F. Martin et al., "Surface pre-coating of talc particles by carboxyl methyl cellulose adsorption: study of adsorption and consequences on surface properties and settling rate," Colloids and Surfaces A, vol. 272, no. 3, pp. 211-219, 2006. 
[25] J. Alam MD, "Effect of metal concentration on shape and composition changes in gold-silver," Bimetallic Systems: Noto-are 15542466: Chemical technology, 2013, https://www .notoare.com/index.php/index/explorer/getPDF/15542466.

[26] S.-A. Riyajan and J. T. Sakdapipanich, "Development of neem capsule via glutaraldehyde crosslinked sodium alginate capsules with natural rubber coating its for its control release," Polymer Bulletin, vol. 63, no. 4, pp. 609-622, 2009.

[27] A. Anitha, S. Maya, N. Deepa et al., "Efficient water soluble O-carboxymethyl chitosan nanocarrier for the delivery of curcumin to cancer cells," Carbohydrate Polymers, vol. 83, no. 2, pp. 452-461, 2011. 

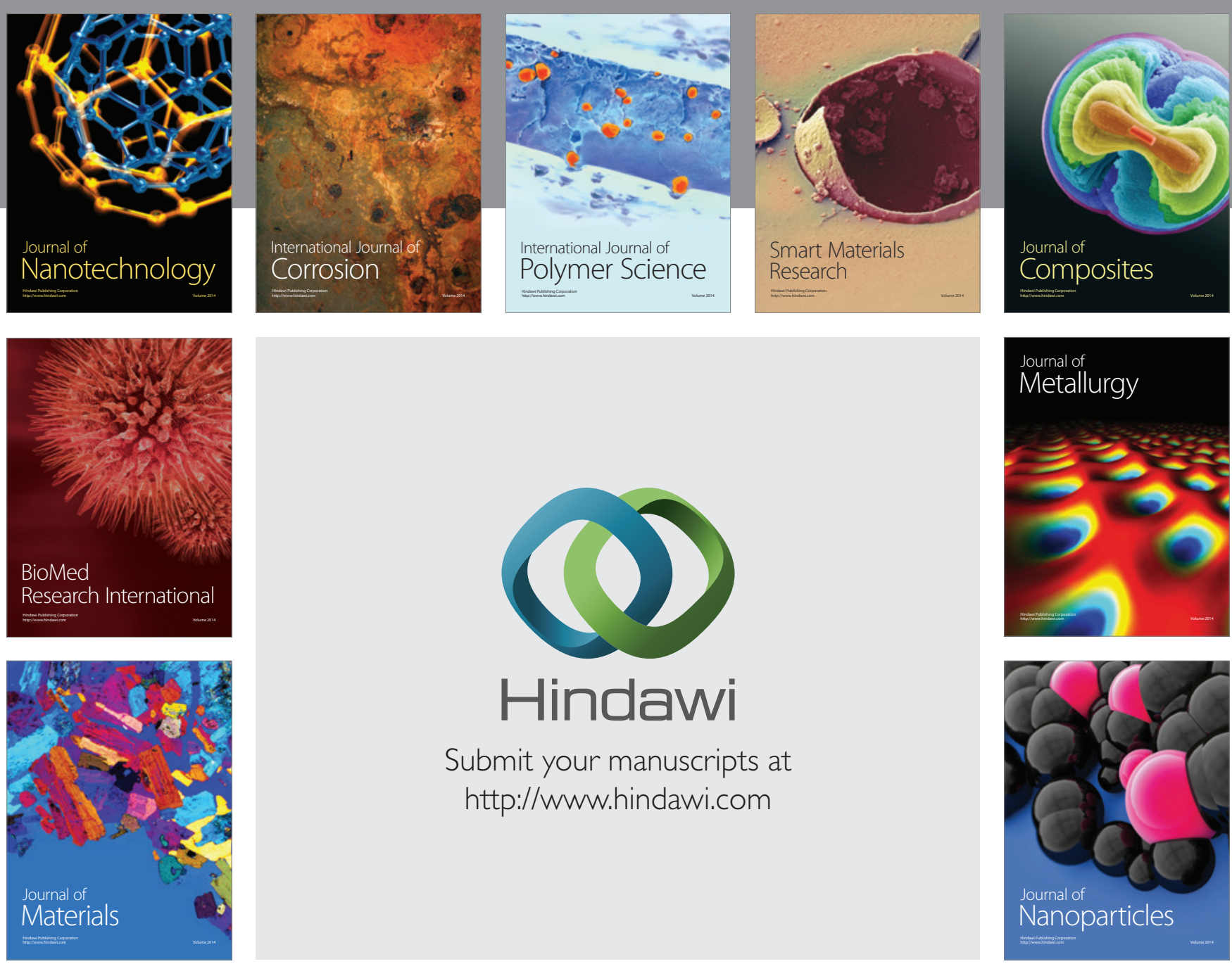

Submit your manuscripts at http://www.hindawi.com
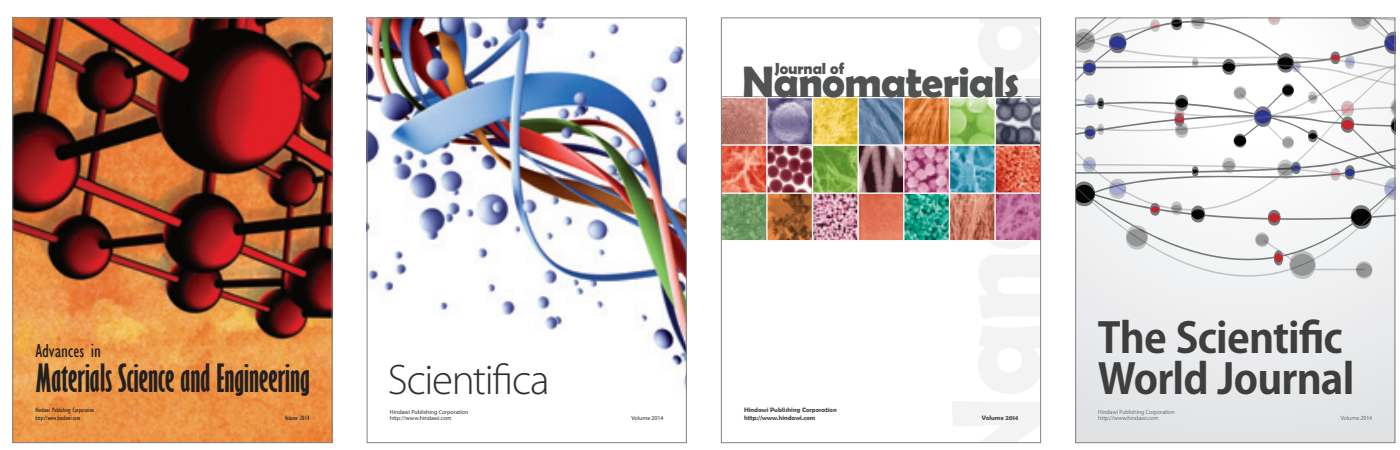

\section{The Scientific World Journal}
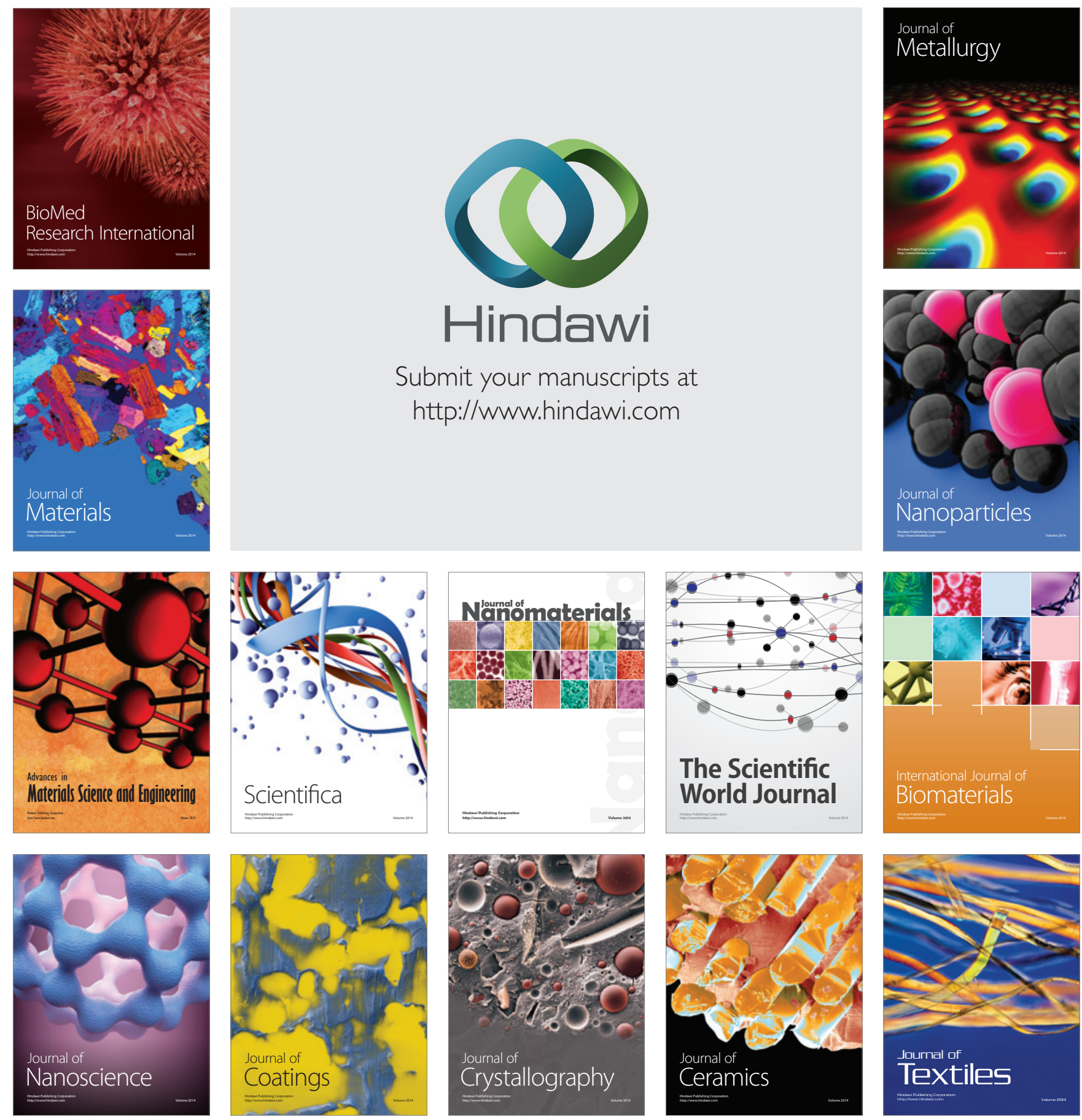